\title{
Diatomáceas epífitas em Galaxaura rugosa (J. Ellis \& Solander) J.V. Lamouroux (Rhodophyta) no Arquipélago de Fernando de Noronha, PE, Nordeste do Brasil ${ }^{1}$
}

Manoel Messias da Silva Costa ${ }^{2,3}$, Enide Eskinazi-Leça ${ }^{2}$, Sonia Maria Barreto Pereira ${ }^{2}$ e Maria Elizabeth Bandeira-Pedrosa ${ }^{2}$

Recebido em 17/04/2008. Aceito em 23/10/2008

RESUMO - (Diatomáceas epífitas em Galaxaura rugosa (J. Ellis \& Solander) J.V. Lamouroux (Rhodophyta) no Arquipélago de Fernando de Noronha, PE, Nordeste do Brasil). Exemplares de Galaxaura rugosa (J. Ellis \& Solander) J.V. Lamouroux foram coletados nos meses de junho/2006 e junho/2007, em três localidades do Arquipélago de Fernando de Noronha (Atalaia, Porto e Cagarras), com o objetivo de identificar a flora das diatomáceas epífitas que habita o talo da alga. Foram identificados 52 táxons distribuídos nas classes: Coscinodiscophyceae (19\%), Fragilariophyceae (21\%) e Bacillariophyceae (60\%) denotando uma dominância de indivíduos com simetria bilateral, os quais corresponderam a $81 \%$ da flora identificada. As seguintes espécies caracterizaram a estrutura florística das diatomáceas, pois foram encontradas em mais de 70\% das amostras analisadas: Amphora sp., Biddulphia biddulphiana (J.E. Smith) Boyer, Cocconeis scutellum Ehrenberg, Diploneis bombus Ehrenberg, Grammatophora marina (Lyngbye) Kützing, Mastogloia binotata (Grunow) Cleve, Navicula longa Grunow, Nitzschia sp., Psammodiscus nitidus (Gregory) Round in Mann, Rhabdonema adriaticum Kützing, Trachyneis aspera (Ehrenberg) Cleve e Tryblionella coarctata (Grunow) Mann. A diversidade específica variou entre média à alta, com os menores valores correspondendo aos florescimentos de Amphora sp. (49,3\%), Mastogloia binotata (42,1\%) e Nitzschia sp. $(62,5 \%)$.

Palavras-chave: abundância, distribuição espaço-temporal, região tropical, riqueza

ABSTRACT - (Epiphytic diatoms on Galaxaura rugosa (J. Ellis \& Solander) J.V. Lamouroux (Rhodophyta) in Fernando de Noronha Archipelago, Pernambuco State, Northeast Brazil). Specimens of Galaxaura rugosa (J. Ellis \& Solander) J.V. Lamouroux were collected in June 2006 and June 2007 at three localities in Fernando de Noronha Archipelago (Atalaia, Porto and Cagarras), aiming to identify the epiphytic diatom flora that inhabits the algae thallus. A total of 52 taxa were identified, distributed in the classes Coscinodiscophyceae (19\%), Fragilariophyceae $(21 \%)$ and Bacillariophyceae $(60 \%)$ with $81 \%$ dominance of individuals with pinnate symmetry. The following species characterized the floristic diatom structure being considered very frequent: Amphora sp., Biddulphia biddulphiana (J.E. Smith) Boyer, Cocconeis scutellum Ehrenberg, Diploneis bombus Ehrenberg, Grammatophora marina (Lyngbye) Kützing, Mastogloia binotata (Grunow) Cleve, Navicula longa Grunow, Nitzschia sp., Psammodiscus nitidus (Gregory) Round in Mann, Rhabdonema adriaticum Kützing, Trachyneis aspera (Ehrenberg) Cleve e Tryblionella coarctata (Grunow) Mann. Specific diversity was médium to high, with lower values for the blooming of Amphora sp. (49.3\%), Mastogloia binotata (42.1\%) and Nitzschia sp. (62.5\%).

Key words: abundance, richness, spatial and temporal distribution, tropical region

\section{Introdução}

As diatomáceas constituem um grupo biológico bastante comum por todo o planeta e são especialmente importantes nos oceanos, onde se estima que contribuam com cerca de 45\% da produção primária oceânica (Mann 1999). Algumas são exclusivamente planctônicas, outras podem viver fixas às rochas ou epífitas sobre algas macroscópicas bentônicas e fanerógamas marinhas (Navarro et al. 1989).

As diatomáceas epífitas representam especial papel nos ambientes marinhos costeiros, sendo responsáveis por grande parte da produção primária, apresentando uma velocidade de produção muitas vezes maior que a das plantas as quais estão aderidas, tornando-se, assim, responsáveis pela transferência de energia sintetizada para os níveis tróficos seguintes (Moreira Filho \& Teixeira 1963).

A comunidade epífita forma uma verdadeira "floresta" que serve de refúgio e alimento para pequenos invertebrados, sendo possível que várias formas vivam na dependência direta desta microvegetação algácea (Virnstein 1987). Segundo Rivera (1973), as macroalgas marinhas constituem excelentes substratos para o desenvolvimento das diatomáceas epífitas e as fanerógamas marinhas também têm sido consideradas bons substratos (McIntire \& Moore 1977; Moreira Filho \& Valente-Moreira 1980).

A maioria das pesquisas com diatomáceas epífitas em macrófitas marinhas abordam os aspectos qualitativos da flora, com considerações sobre a composição e a variação florística entre diversos hospedeiros, já existindo um conhecimento aprofundado sobre as relações hospedeiros/ epífitas (Edelstein \& Komarovsky 1961; Takano 1961; 1962; Edsbagge 1968; Rivera 1973; Main \& McIntire 1974; Navarro 1982; Wah \& Wee 1988; Navarro et al. 1989; Nagumo \& Hara 1990; Bergey et al. 1995; Ruesink 1998; SiqueirosBeltrones \& Castrejón 1999; Siqueiros-Beltrones et al. 2002; Siqueiros-Beltrones et al. 2005; Frankovich et al. 2006).

A exemplo do que ocorre no mundo, as pesquisas realizadas no Brasil também estão direcionadas ao conhecimento florístico das populações de áreas costeiras, conhecendo-se a flora epífita que ocorrem em clorofíceas, feofíceas e rodofíceas (Moreira Filho 1959; Kutner 1961; Moreira Filho 1966; Moreira Filho et al. 1977-1978; Moreira Filho \& Valente-Moreira 1980; 1981; Valente-Moreira \& Moreira Filho 1980; Buselato-Toniolli 1986; Azevedo 1999; Azevedo \& Cutrim 1999; 2000).

\footnotetext{
Parte da Dissertação de Mestrado do primeiro Autor

2 Universidade Federal Rural de Pernambuco, Departamento de Biologia, Programa de Pós-Graduação em Botânica, Recife, PE, Brasil

3 Autor para correspondência: manoelbiologo@ig.com.br
} 
Em Pernambuco, várias investigações já foram realizadas enfocando a biodiversidade desta microvegetação epífita, porém a maioria aborda as diatomáceas aderidas às folhas de fanerógamas marinhas, destacando-se as contribuições de Laborel-Denguen (1963), Pacobahyba et al. (1993) e Cunha \& Eskinazi-Leça (2003) e de Moura et al. (1993) com raízes e caules de mangues.

Considerando a carência de estudos qualitativos com diatomáceas epífitas em macroalgas marinhas e a necessidade de estudos deste importante grupo de produtores primários no Arquipélago de Fernando de Noronha, o presente trabalho teve como objetivo identificar a composição e a distribuição espaço-temporal da comunidade de diatomáceas que cresce aderida às diversas partes do talo da alga vermelha Galaxaura rugosa, espécie comum no Arquipélago.

\section{Material e métodos}

Área de estudo - O Arquipélago de Fernando de Noronha é de origem vulcânica, situado no Atlântico Sul equatorial ( $3^{\circ} 51^{\prime} 03^{\prime \prime}-3^{\circ} 58^{\prime} 55^{\prime \prime}$; $32^{\circ} 22^{\prime} 29^{\prime}$ '-32'29'40'W), distando 345 km do Cabo de São Roque (Rio Grande do Norte) e $545 \mathrm{~km}$ da cidade de Recife (Pernambuco) (Fig. 1). O arquipélago está ligado politicamente desde 1988 ao Estado de Pernambuco, tendo sido criado, nessa época, o Parque Nacional Marinho de Fernando de Noronha. Constitui-se de uma ilha principal que lhe dá o nome, com cerca de $16,4 \mathrm{~km}^{2}$, que representa $91 \%$ da área do arquipélago, e ao seu redor encontram-se 20 ilhotas (Almeida 2002; Villaça et al. 2006). O clima é tropical do tipo Awi segundo sistema Köppen. A temperatura média anual do ar fica em torno de $27{ }^{\circ} \mathrm{C}$ e da água de $24^{\circ} \mathrm{C}$, salinidade de 36 e precipitação anual em torno de $1.300 \mathrm{~mm}$. Ocorrem ventos alísios, que têm ações diretas no lado meridional do Arquipélago voltado para o continente africano, que é chamado de "mar de fora" e, devido a isso, possui águas revoltas durante o ano todo. O lado setentrional da ilha voltado para o Atlântico norte, mais protegido dos ventos e correntes, é chamado de "mar de dentro" (Teixeira et al. 2003). As marés são semi-diurnas com amplitude de 3,2 $\mathrm{m}$ durante as marés de sizígia e 2,0 $\mathrm{m}$ nas marés de quadratura (Eston et al. 1986). Durante o ano ocorrem duas estações bem definidas: a chuvosa, de fevereiro a julho, e de estiagem, de agosto a janeiro. A umidade relativa do ar apresenta valores entre $85 \%$ (chuvoso) e $81 \%$ (estiagem).

Descrição do hospedeiro - A Rhodophyta Galaxaura rugosa (Galaxauraceae, Nemaliales, Florideophyceae) é uma alga macroscópica que apresenta o talo com forma ereta e do tipo cilíndrico, com hábito epilítico e parede celular ligeiramente impregnada de carbonato de cálcio. Ramos cilíndricos ocos, com 1 a $3 \mathrm{~mm}$ diâm., e 3 a $8 \mathrm{~cm}$ alt., possuindo ramificações subdicotômicas ao longo do talo, cobertos por filamentos assimiladores longos em verticilos definidos da porção mediana até o ápice. A sua distribuição no Brasil vai desde o litoral Sudeste até o litoral nordestino, inclusive no Arquipélago de Fernando de Noronha (Pereira 2006).

Coleta e análises - As coletas foram realizadas durante duas campanhas (junho/2006 e junho/2007), nas regiões entre-marés e infralitoral das seguintes localidades: Atalaia (Ponto 1), Porto (Ponto 2) e Cagarras (Ponto 3), durante as marés de sizígia (Fig. 1). Na praia de Cagarras foram examinados apenas exemplares coletados em junho/2006, em virtude da ausência da alga durante a coleta de junho/2007. Em cada Ponto, foram coletados vários exemplares de $G$. rugosa, com o auxílio de espátulas para remoção do talo por inteiro. Após a coleta, o material (hospedeiro-epífito) foi preservado em solução com formol a $4 \%$, neutralizado com bórax a 1\%, e transportado ao Laboratório de Ficologia (LABOFIC) do Programa de Pós-Graduação em Botânica da Universidade Federal Rural de Pernambuco.

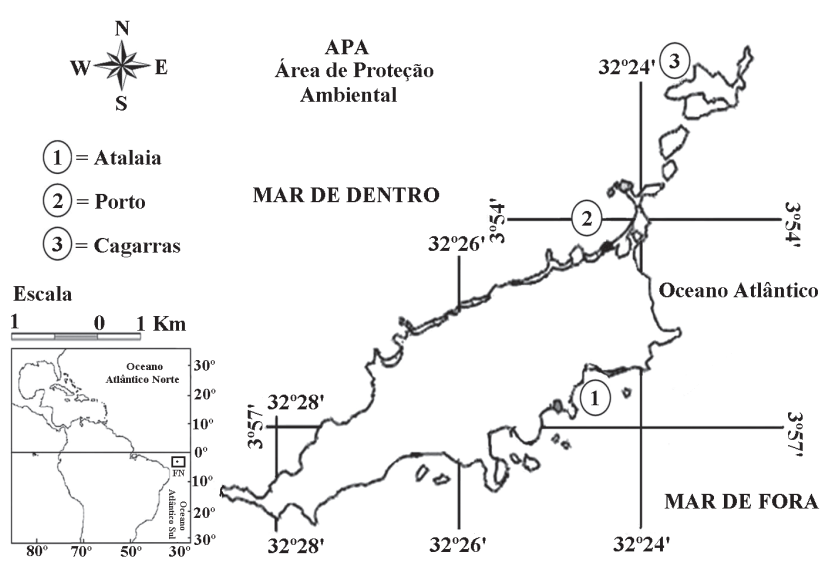

Figura 1. Localização dos Pontos de coleta - (1) Atalaia, (2) Porto e (3) Cagarras no Arquipélago de Fernando de Noronha, PE, Nordeste do Brasil (adaptado de Almeida 2002).

De cada coleta, foram selecionados aleatoriamente três exemplares, que foram lavados com água destilada para remoção do sedimento e, em seguida, os exemplares divididos em três porções iguais (apical, mediana e basal) de acordo com o seu comprimento total.

Os fragmentos foram submetidos à oxidação direta com hipoclorito de sódio a $1 \%$, por 24 horas, para separação das diatomáceas do hospedeiro. $\mathrm{O}$ material oxidado passou por sucessivas lavagens (seis lavagens) com água destilada em centrífuga, a $1.000 \mathrm{rpm}$, por aproximadamente 2 minutos. Do material oxidado foram confeccionadas 15 lâminas permanentes de acordo com o método descrito por Ferrario et al. (1995). As lâminas permanentes foram depositadas no Herbário Prof. Vasconcelos Sobrinho-PEUFR. Para a identificação dos táxons foi utilizada a seguinte literatura: Van Heurk (1896), Peragallo \& Peragallo (1897-1908), Hustedt (1930; 1937-1938; 1961), Cupp (1943), Moreira Filho et al. (1977-1978), Takano (1961; 1962), Ricard (1987), Moreno et al. (1996). Utilizou-se o sistema de classificação de Round et al. (1990) para o enquadramento dos táxons.

Após a identificação, foram realizadas contagens das valvas presentes em cada lâmina e, a partir daí, calculada a abundância relativa de cada táxon, através da fórmula: $\mathrm{A}=\mathrm{N} \times 100 / \mathrm{n}$ onde, $\mathrm{N}=\mathrm{n}^{\circ}$ de espécies na lâmina; $\mathrm{n}=\mathrm{n}^{\circ}$ total de espécies, sendo estabelecidos os seguintes critérios: dominante $=>50 \%$; abundante $=50-30 \%$; pouco abundante $=30-10 \%$ e rara $=\leq 10 \%$.

A freqüência de ocorrência foi calculada a partir do número de vezes em que cada táxon ocorreu nas lâminas analisadas, através da fórmula: $\mathrm{F}=\mathrm{P} \times 100 / \mathrm{p}$ onde, $\mathrm{P}=\mathrm{n}^{\circ}$ de lâminas contendo a espécie; $\mathrm{p}=\mathrm{n}^{\circ}$ total de lâminas examinadas, sendo estabelecidos os seguintes critérios: muito freqüente $=>70 \%$ das lâminas; freqüente $=70-40 \%$; pouco freqüente $=40-20 \%$ e esporádica $=\leq 20 \%$ ).

A diversidade específica foi calculada segundo Shannon (1948). Os valores acima de 3 bits.cel ${ }^{-1}$ foram considerados como de alta diversidade, 2 bits.cel $^{-1}$ de média diversidade e valores abaixo de 1 bits.cel ${ }^{-1}$ de baixa diversidade. A eqüitabilidade foi calculada a partir do índice de Shannon, adotando-se os valores maiores que 0,50 como sendo de alta eqüitabilidade e menores, considerados de baixa eqüitabilidade. Para os dados estatísticos, aplicou-se análise de variância (ANOVA) para os pontos de coleta e teste t Student para os períodos estudados, nível de significância $(\mathrm{p}<0,05)$ Zar (1999).

Foi confeccionada uma matriz, considerando-se a presença e ausência das diatomáceas epífitas nos exemplares de G. rugosa coletados nos meses de junho/2006 e junho/2007, nas praias de Atalaia, Porto e Cagarras. A matriz de similaridade foi calculada pelo coeficiente de Jaccard, e a partir destes dados foi construído o dendrograma, utilizando-se o Programa NTSYSpc 2.01t software (Rohlf 2000), sendo analisado 52 OTUs (Unidades Taxonômicas Operacionais) a nível de 70\% (Jaccard 1901). 


\section{Resultados e discussão}

Na rodofíta Galaxaura rugosa foram identificadas 52 espécies de diatomáceas distribuídas nas classes: Coscinodiscophyceae (10 espécies), Fragilariophyceae (11 espécies) e Bacillariophyceae (31 espécies), denotando uma dominância de indivíduos com simetria bilateral, os quais corresponderam a $81 \%$, quando somados os táxons das classes Fragilariophyceae (21\%) e Bacillariophyceae (60\%) (Fig. 2).

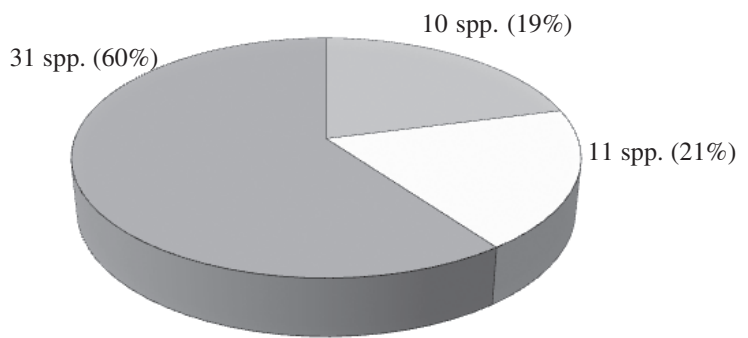

Figura 2. Contribuição relativa das classes de diatomáceas epífitas identificadas na rodofícea Galaxaura rugosa (J. Ellis \& Solander) J.V. Lamouroux, coletada no Arquipélago de Fernando de Noronha, PE, Nordeste do Brasil, nos meses de junho/2006 e junho/2007 ( $\square=$ Coscinodiscophyceae; $\square=$ Fragilariophyceae, = Bacillariophyceae).

De acordo com Main \& McIntire (1974) e Wetherbee et al. (1998), as diatomáceas de simetria bilateral possuem estruturas próprias para a produção de substâncias mucilaginosas e, por este motivo, podem melhor se aderir a seus hospedeiros, através de pedúnculos curtos ou longos, ou em camadas de uma matriz gelatinosa. A maior representatividade desses indivíduos em outras macrófitas ocorrentes no litoral de Pernambuco também tem sido documentada (Moura et al. 1993; Pacobahyba et al. 1993; Cunha \& Eskinazi-Leça 2003; Eskinazi-Leça et al. 2003). No caso de $G$. rugosa, a presença dominante de diatomáceas capazes de permanecerem mais tempo aderidas aos hospedeiros, é um fato justificável, considerando-se que os exemplares foram coletados em ambiente insular, sob ação direta de ventos alísios e de correntes marinhas durante maior parte do ano (Teixeira et al. 2003).

No Arquipélago de Fernando de Noronha, os maiores valores de riqueza ocorreram nas praias de Porto e Cagarras, locais que sofrem menor influência da ação dos ventos e das correntes marinhas, enquanto que a menor riqueza foi detectada em exemplares coletados na praia de Atalaia, localizada na parte oriental do Arquipélago, sujeita a maior movimentação da água (Fig. 3). Pesquisas realizadas por Navarro et al. (1989), sobre diatomáceas aderidas a diversos substratos em Porto Rico, revelaram o mesmo tipo de distribuição, ou seja, maior riqueza de espécies em substratos existentes em locais com movimento moderado da água, enquanto a menor diversidade foi encontrada em hospedeiros que habitavam locais com forte ação das correntes.

Para Main \& McIntire (1974) e Navarro (1983), as diferenças na estrutura das comunidades de diatomáceas epífitas podem ocorrer quando os hospedeiros crescem em diferen-

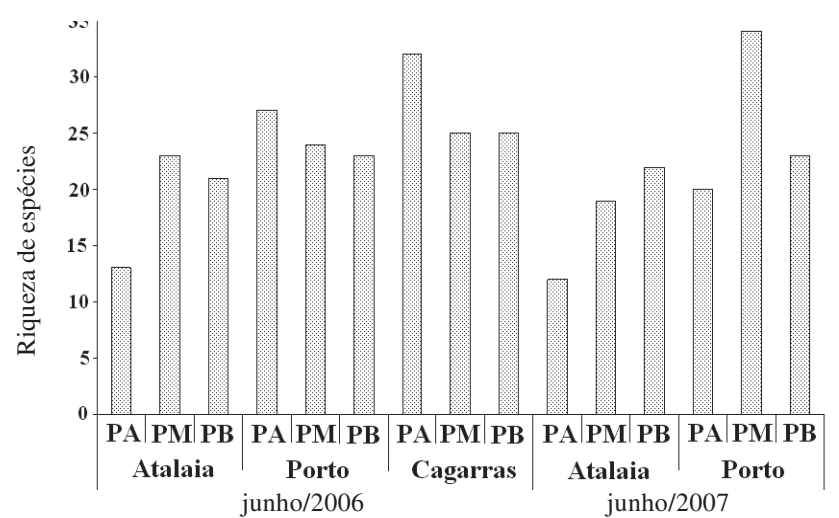

Figura 3. Riqueza de espécies de diatomáceas epífitas, identificadas na rodofícea Galaxaura rugosa (J. Ellis \& Solander) J.V. Lamouroux, coletada no Arquipélago de Fernando de Noronha, PE, Nordeste do Brasil, nos meses de junho/2006 e junho/2007 - (PA) porção apical, (PM) porção mediana, (PB) porção basal.

tes locais de exposição à luz, como também por estarem sujeitas às diferenças de direção e velocidade das correntes marinhas, pois o tipo de movimentação da água pode selecionar a flora associada de acordo com seus meios de fixação.

As 52 espécies de diatomáceas identificadas apresentaram uma distribuição pouco uniforme ao longo do talo da alga, tendo ocorrido menor riqueza (12 espécies) na porção apical de exemplares de G. rugosa coletados na praia de Atalaia (junho de 2007) e maior riqueza de espécies, observadas na porção mediana de exemplares coletados na praia do Porto (34 espécies), no mês de junho de 2007 (Fig. 3, Tab. 1). Mesmo assim, não foram observados diferenças significativa entre pontos de coleta $(\mathrm{p}=0.097680)$ e nem entre os meses amostrados (junho/2006 e junho/2007) $(\mathrm{p}=0.197753)$.

Algumas espécies ocorreram com percentuais significativos de abundância como Nitzschia sp. $(62,5 \%)$ e Amphora sp. (49,3\%) na praia de Atalaia, e Mastogloia binotata $(42,1 \%)$ na praia do Porto (Tab. 1), influenciando os índices de diversidade específica, a qual variou entre 1,86 bits.cel $^{-1}$ na praia de Atalaia e 3,69 bits.cel $^{-1} \mathrm{em} \mathrm{Cagarras,}^{-1}$ ambos no mês de junho de 2006 (Fig. 4,Tab. 1). Porém, os valores de diversidade específica, de um modo geral, apresentaram uma variação de média à alta, em torno de $87 \%$ das amostras analisadas. Em relação à eqüitabilidade, as amostras mostraram uma distribuição eqüitativa das espécies, com valores acima a 0,50 (Fig. 4).

Para McIntire \& Moore (1977) e Ferreira \& Seeliger (1985), a baixa diversidade específica é uma característica marcante na comunidade de diatomáceas epífitas cujo padrão de diversidade é influenciado diretamente por poucas espécies que contribuem em número de indivíduos na caracterização da estrutura da comunidade. Este padrão tem sido confirmado em diversos hospedeiros analisados quer sejam macroalgas ou fanerógamas marinhas (Navarro 1982; Navarro 1983; Pacobahyba et al. 1993; Cunha \& Eskinazi-Leça 2003).

No caso de Galaxaura rugosa, a diversidade específica mostrou uma tendência diferente de outros hospedeiros já analisados no litoral de Pernambuco, com índices que variaram entre média à alta diversidade biológica. Da mesma 
Tabela 1. Distribuição percentual das espécies de diatomáceas epífitas, no talo da rodofícea Galaxaura rugosa (J. Ellis \& Solander) J.V. Lamouroux, coletada no Arquipélago de Fernando de Noronha, PE, Nordeste do Brasil, nos meses de junho/2006 e junho/2007 - (PA) porção apical, (PM) porção mediana, (PB) porção basal, (FO) frequiência de ocorrência, $(\mathrm{MF})$ muito freqüente, $(\mathrm{F})$ freqüente, $(\mathrm{PF})$ pouco freqüente, $(\mathrm{E})$ esporádico; - = Dados não existentes.

\begin{tabular}{|c|c|c|c|c|c|c|c|c|c|c|c|c|c|c|c|c|}
\hline \multirow[t]{3}{*}{ Espécies/Períodos } & \multicolumn{9}{|c|}{ junho/2006 } & \multicolumn{7}{|c|}{ junho/2007 } \\
\hline & \multicolumn{3}{|c|}{ Atalaia } & \multicolumn{3}{|c|}{ Porto } & \multicolumn{3}{|c|}{ Cagarras } & \multicolumn{3}{|c|}{ Atalaia } & \multicolumn{3}{|c|}{ Porto } & \\
\hline & PA & $\mathrm{PM}$ & PB & PA & $\mathrm{PM}$ & PB & PA & PM & PB & PA & $\mathrm{PM}$ & $\mathrm{PB}$ & PA & PM & $\mathrm{PB}$ & FO \\
\hline
\end{tabular}

\section{DIVISÃO BACILLARIOPHYTA \\ CLASSE COSCINODISCOPHYCEAE}

Biddulphia biddulphiana (J.E. Smith) Boye

B. tridens (Ehrenberg) Ehrenberg

Cyclotella sp.

Eunotogramma laeve Grunow in Cleve et Möller

Odontella dubia (Brightwell) Chávez y Baumgartner-

Paralia sulcata (Ehrenberg) Cleve

Psammodiscus nitidus (Gregory) Round in Mann

Terpsinö̈ musica Ehrenberg

Triceratium favus Ehrenberg

T. pentacrinus (Ehrenberg) Wallich

CLASSE FRAGILARIOPHYCEAE

Climacosphenia moniligera Ehrenberg

Delphineis karstenii (Boden) Fryxell

Grammatophora marina (Lyngbye) Kützing

G. oceanica (Ehrenberg) Grunow

Plagiogramma pulchellum Greville

Podocystis adriatica Kützing

P. guadalupensis Ricard

P. spathulata (Shadbolt) Van Heurk

Rhabdonema adriaticum Kützing

Rhaphoneis castracanei Grunow \& Wood

Synedra sp.

CLASSE BACILLARIOPHYCEAE

Achnanthes brevipes Agardh

Amphora angusta Gregory

A. bigibba Hagelstein

A. macilenta Gregory

Amphora sp.

Campylodiscus innominatus Ross \& Abdin

Cocconeis heteroidea Hantzsch

C. molesta var. crucifera Grunow

C. scutellum Ehrenberg

Diploneis bombus Ehrenberg

D. crabro (Ehrenberg) Ehrenberg

D. vacillans (Schmidt) Cleve

D. weissflogii (Schmidt) Cleve

Entomoneis alata (Ehrenberg) Ehrenberg

Lyrella lyra (Ehrenberg) Karayeira

Mastogloia binotata (Grunow) Cleve

M. quinquecostata Grunow

M. minuta Greville

M. splendida (Gregory) Cleve

Navicula longa Grunow

Nitzschia sp.

$\begin{array}{lllllllll}0,8 & 0,2 & 0,6 & 5,0 & 3,0 & 2,1 & 3,0 & 0,7 & 1,9 \\ - & - & - & - & - & - & 1,1 & - & - \\ - & - & - & - & - & - & - & - & - \\ - & - & - & - & - & - & - & - & - \\ \text { ner- } & - & - & 0,7 & 0,6 & - & 0,3 & 0,4 & 0,2 \\ - & - & - & 0,1 & - & - & - & - & - \\ - & 2,0 & 0,6 & 2,1 & 1,2 & 1,7 & 1,2 & 1,4 & 0,7 \\ - & - & - & - & - & - & - & - & - \\ - & - & - & - & - & - & - & 0,2 & - \\ - & 0,5 & - & - & - & - & - & - & -\end{array}$

$\begin{array}{llllll}0,5 & 1,0 & 3,6 & 5,5 & 2,8 & \mathrm{MF}\end{array}$

$\begin{array}{llllll}- & - & - & - & - & \mathrm{E} \\ - & - & 2,3 & 1,3 & 0,2 & \mathrm{E} \\ - & - & - & 0,8 & - & \mathrm{E} \\ - & - & 1,0 & 1,8 & 0,4 & \mathrm{~F} \\ - & - & - & - & - & \mathrm{E} \\ - & 5,4 & 0,6 & 2,6 & 2,4 & \mathrm{MF} \\ - & - & 1,6 & 0,3 & 0,2 & \mathrm{E} \\ - & - & - & 0,3 & - & \mathrm{E} \\ - & - & - & - & - & \mathrm{E}\end{array}$

$\begin{array}{lllllllllllllllll}- & - & - & - & - & - & - & - & - & 1,6 & 0,5 & - & - & 1,3 & 0,2 & \mathrm{PF} \\ - & 0,2 & - & - & - & 0,3 & - & - & 0,2 & - & - & - & - & - & - & \mathrm{E} \\ 1,5 & 7,5 & 4,0 & 4,7 & 0,7 & 2,0 & 13,2 & 5,3 & 6,9 & 4,6 & 1,9 & 0,4 & 0,3 & 0,8 & - & \mathrm{MF} \\ - & 0,2 & - & - & 0,3 & - & 0,9 & 0,4 & 1,2 & - & - & 0,4 & - & - & - & \mathrm{PF} \\ - & - & - & - & - & - & - & - & - & - & - & - & 2,9 & 1,0 & 1,1 & \mathrm{E} \\ - & 1,4 & 0,6 & 1,3 & 0,7 & - & 13,2 & 4,4 & 6,2 & - & 0,5 & 0,4 & - & 0,3 & - & \mathrm{F} \\ - & - & - & - & 0,1 & - & - & - & - & - & - & - & - & - & - & \mathrm{E} \\ - & - & - & - & - & - & 0,3 & - & - & - & - & - & - & - & - & \mathrm{E} \\ 0,8 & 0,7 & 0,9 & 1,0 & - & 0,5 & 0,1 & 0,9 & 0,7 & - & 0,5 & 0,4 & 1,0 & 0,3 & - & \mathrm{MF} \\ - & - & - & - & - & - & - & 0,2 & - & - & - & - & - & - & - & \mathrm{E} \\ - & - & - & 0,6 & - & - & 0,7 & 0,5 & - & - & 0,5 & 0,4 & 0,3 & 0,3 & - & \mathrm{F}\end{array}$

Petroneis humerosa (Brébisson) Stickle and Mann

Plagiodiscus martensianus Grunow \& Eulenstein

Pleurosigma formosum Wm. Smith

Psammodictyon panduriforme Gregory

Rhopalodia musculus (Kützing) Müller

Surirella fastuosa Ehrenberg

Trachyneis aspera (Ehrenberg) Cleve

Tryblionella coarctata (Grunow) Mann

T. hungarica (Grunow) Mann

T. granulata (Grunow) Mann

Total

Riqueza de espécies

$\begin{array}{llllllllllllllll}- & - & 0,9 & - & - & 0,3 & 0,1 & 0,4 & - & 7,8 & - & 1,3 & - & 1,8 & 2,4 & \text { F } \\ - & 0,2 & 0,6 & 2,1 & 0,6 & - & 5,7 & - & 6,5 & - & - & 0,4 & 0,6 & 0,8 & 0,4 & \text { F } \\ - & - & - & - & - & - & - & 0,2 & 0,2 & - & - & - & - & - & 0,9 & \text { E } \\ 7,2 & - & - & - & - & - & 0,8 & - & - & 1,6 & 2,3 & - & - & 0,3 & 0,9 & \text { PF }\end{array}$

$\begin{array}{lcc}- & - & - \\ 9,4 & 25,4 & 5,9\end{array}$

$6,1 \quad 28,5 \quad 17,2 \quad 35,1-35,2-14$

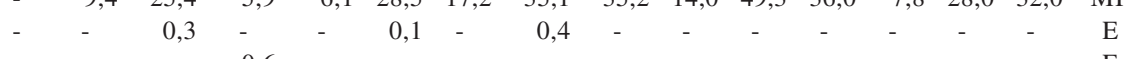

$\begin{array}{lllllllllllllll}- & - & - & 0,6 & - & - & - & - & - & - & - & - & - & - & - \\ -\end{array}$

$\begin{array}{lllllllllllllllll}- & 0,5 & 0,3 & 0,7 & - & 1,1 & 0,7 & 1,8 & 0,5 & 1,6 & 0,9 & 0,4 & - & 0,5 & - & \mathrm{MF}\end{array}$

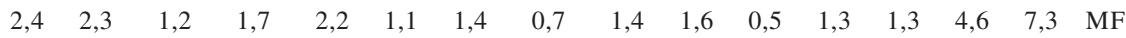

$\begin{array}{llllllllllllllll}- & 0,7 & 0,9 & 0,7 & 0,1 & 0,6 & 0,3 & - & - & - & - & 1,0 & - & - & 0,2 & F \\ - & 0,7 & 0,9 & - & - & - & - & - & - & - & 3,3 & 1,0 & 0,3 & 1,3 & 1,7 & F\end{array}$

$\begin{array}{llllllllllllllll}- & 0,7 & 0,9 & - & - & - & - & - & - & - & 3,3 & 1,0 & 0,3 & 1,3 & 1,7 & F \\ - & - & - & 0,4 & 0,4 & - & - & - & - & - & - & - & 5,2 & 0,8 & - & \text { PF } \\ - & - & - & 0,1 & 0,1 & 0,3 & 0,4 & 0,2 & 0,7 & - & - & - & - & - & - & P F\end{array}$

$\begin{array}{llllllllllllllll}- & - & - & - & - & - & - & - & - & - & - & - & - & 0,3 & - & \mathrm{E} \\ 0,8 & 0,7 & 1,4 & 28,6 & 30,0 & 8,1 & 16,5 & 7,4 & 5,5 & - & 2,3 & 4,9 & 42,1 & 12,0 & 13,9 & \mathrm{MF}\end{array}$

$\begin{array}{cccccccccccccccc}- & 0,2 & 0,6 & - & - & 2,3 & 0,4 & - & - & 11,0 & 0,5 & 0,4 & - & - & - & F \\ - & - & - & 8,0 & 0,7 & 2,3 & 0,3 & - & 0,5 & - & - & - & - & 0,5 & - & P F\end{array}$

$\begin{array}{llllllllllllllll}0,8 & - & - & & 1,3 & 0,7 & 0,1 & - & 1,1 & 1,1 & 1,6 & - & - & - & - & -\end{array}$

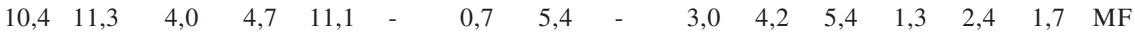

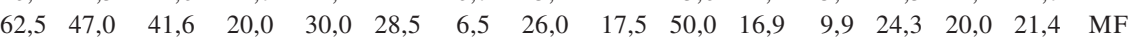

Diversidade específica (bits.cel ${ }^{-1}$ )

Eqüitabilidade

$\begin{array}{lllllllllllllllll}- & - & - & - & - & - & 0,1 & - & 0,2 & - & - & - & - & - & - & \mathrm{E} \\ - & 0,5 & - & - & - & 0,5 & 0,3 & - & 1,1 & - & - & - & - & - & - & \mathrm{PF} \\ 3,2 & 1,2 & 2,3 & 1,4 & - & 1,3 & 0,1 & - & - & - & 9,4 & 18,0 & - & 0,3 & - & \mathrm{F} \\ - & - & - & 1,1 & 0,9 & 0,6 & 6,0 & 1,2 & 3,3 & - & - & 3,1 & - & 1,0 & 2,6 & \mathrm{~F} \\ - & 1,2 & 1,4 & 0,3 & 0,1 & - & 0,3 & - & - & 1,6 & - & - & - & 0,3 & - & \mathrm{F} \\ 0,8 & 2,0 & 4,3 & 0,1 & 0,6 & 2,0 & 3,5 & - & 0,9 & - & 2,3 & 5,4 & 0,6 & 2,5 & 1,9 & \mathrm{MF} \\ 8,0 & 9,4 & 7,2 & 6,4 & 6,4 & 11,2 & 2,2 & 4,6 & 5,1 & - & 2,3 & 3,1 & 2,3 & 3,4 & 3,2 & \mathrm{MF} \\ - & - & - & - & 3,0 & 4,5 & 2,4 & 0,9 & 1,9 & - & - & - & - & - & 0,9 & \mathrm{PF} \\ 0,8 & - & - & 0,4 & 0,4 & - & 0,1 & 0,2 & 0,4 & - & - & - & 0,6 & 1,8 & 1,3 & \mathrm{~F}\end{array}$

$\begin{array}{lllllllllllllll}100,0 & 100,0 & 100,0 & 100,0 & 100,0 & 100,0 & 100,0 & 100,0 & 100,0 & 100,0 & 100,0 & 100,0 & 100,0 & 100,0 & 100,0\end{array}$

$\begin{array}{lllllllllllllll}13 & 23 & 21 & 27 & 24 & 23 & 32 & 25 & 25 & 12 & 19 & 22 & 20 & 34 & 23\end{array}$

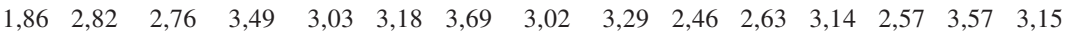

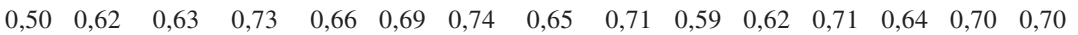


forma, se observou que as espécies identificadas foram predominantemente eqüitativas nas coletas realizadas.

Os altos índices de diversidade específica em G. rugosa coletada no Arquipélago de Fernando de Noronha sugerem que a grande movimentação das águas não chega a ser um fator de influência na distribuição das espécies, facilitando, por outro lado, a grande fixação de espécies de simetria bilateral, que têm maior poder de adesão.

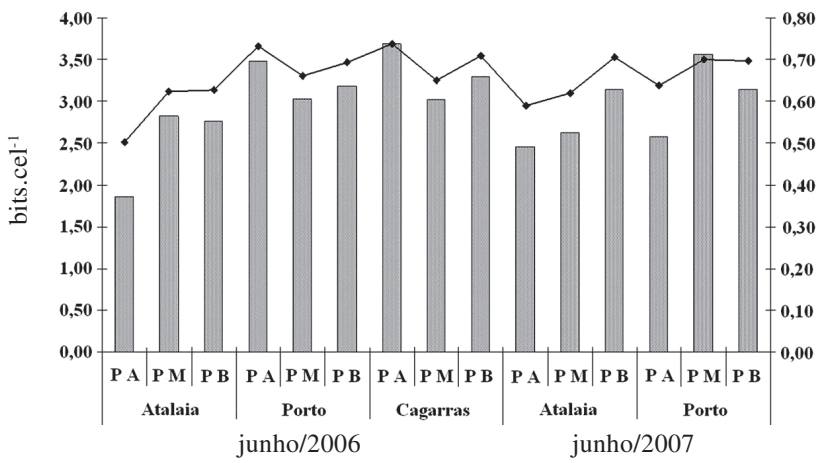

Figura 4. Índice de Diversidade específica (bits.cel ${ }^{-1}$ ) e Eqüitabilidade das espécies de diatomáceas epífitas, identificadas na rodofícea Galaxaura rugosa (J. Ellis \& Solander) J.V. Lamouroux, coletada no Arquipélago de Fernando de Noronha, PE, Nordeste do Brasil, nos meses de junho/2006 e junho/2007 - (PA) porção apical, (PM) porção mediana, $(\mathrm{PB})$ porção basal. = = Diversidade específica; $-\bullet-=$ Eqüitabilidade.
As seguintes espécies caracterizaram a estrutura florística das diatomáceas epífitas, por estarem presentes em mais $70 \%$ das amostras analisadas e, assim, consideradas espécies muito freqüentes: Amphora sp., Biddulphia biddulphiana, Cocconeis scutellum, Diploneis bombus, Grammatophora marina, Mastogloia binotata, Navicula longa, Nitzschia sp., Psammodiscus nitidus, Rhabdonema adriaticum, Trachyneis aspera e Tryblionella coarctata, representando $23 \%$ de todos os táxons analisados. Os demais se enquadraram nas seguintes categorias: freqüentes $(25 \%)$, pouco freqüentes $(15 \%)$ e esporádicos $(37 \%)$ (Tab. 1).

O dendrograma de similaridade confirmou a formação de oito agrupamentos: 1) grupo principal, formado por espécies com freqüência de ocorrência maior que $70 \%$, subdividido em dois subgrupos, o primeiro, formado pelas espécies Diploneis bombus e Nitzschia sp., que estiveram presentes em todas as porções do talo e em todos os locais de coleta, e o segundo subgrupo, formado por Biddulphia biddulphiana, Mastogloia binotata e Tryblionella coarctata que estiveram ausentes, apenas na porção apical de exemplares coletados na praia de Atalaia, no mês de junho/2007; 2) formado por espécies presentes em duas estações, menos em Atalaia (Odontella dubia e Tryblionella granulata); 3) formado por Entomoneis alata, Mastogloia minuta e Tryblionella hungarica, presentes nas porções

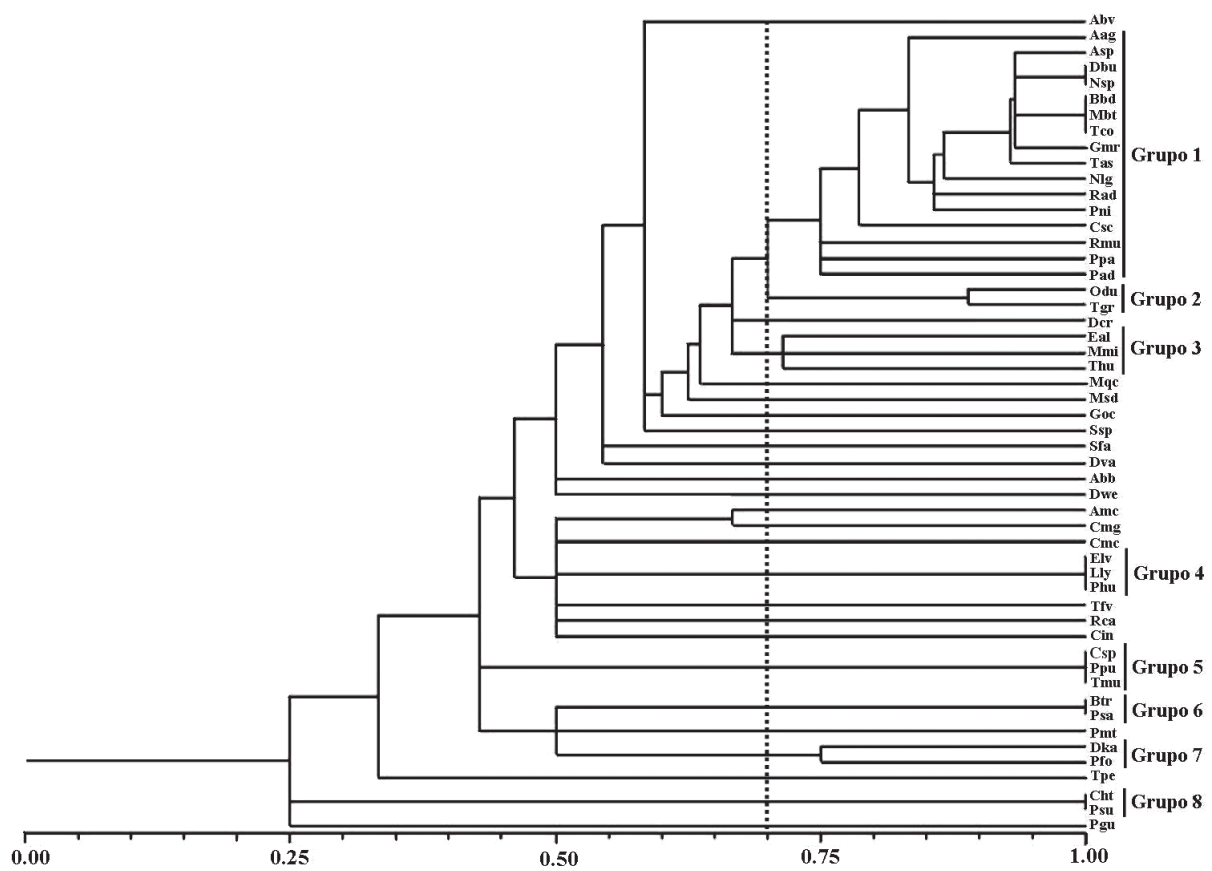

Figura 5. Agrupamentos das espécies de diatomáceas epífitas, identificadas na rodofícea Galaxaura rugosa (J. Ellis \& Solander) J.V. Lamouroux, coletada no Arquipélago de Fernando de Noronha, PE, Nordeste do Brasil, nos meses de junho/2006 e junho/2007 - Abv: Achnanthes brevipes, Aag: Amphora angusta, Asp: Amphora sp., Dbu: Diploneis bombus, Nsp: Nitzschia sp., Bbd: Biddulphia biddulphiana, Mbt: Mastogloia binotata, Tco: Tryblionella coarctata, Gmr: Grammatophora marina, Tas: Trachyneis aspera, Nlg: Navicula longa, Rad: Rhabdonema adriaticum, Pni: Psammodiscus nitidus, Csc: Cocconeis scutellum, Rmu: Rhopalodia musculus, Ppa: Psammodictyon panduriforme, Pad: Podocystis adriatica, Odu: Odontella dubia, Tgr: Tryblionella granulata, Dcr: Diploneis crabro, Eal: Entomoneis alata, Mmi: Mastogloia minuta, Thu: Tryblionella hungarica, Mqc: Mastogloia quinquecostata, Msd: Mastogloia splendida, Goc: Grammatophora oceanica, Ssp: Synedra sp., Sfa: Surirella fastuosa, Dva: Diploneis vacillans, Abb: Amphora bigibba, Dwe: Diploneis weissflogii, Amc: Amphora macilenta, Cmg: Climacosphenia moniligera, Cmc: Cocconeis molesta var. crucifera, Elv: Eunotogramma laeve, Lly: Lyrella lyra, Phu: Petroneis humerosa, Tfv: Triceratium favus, Rca: Rhaphoneis castracanei, Cin: Campylodiscus innominatus, Csp: Cyclotella sp., Ppu: Plagiogramma pulchellum, Tmu: Terpsinö̈ musica, Btr: Biddulphia tridens, Psa: Podocystis spathulata, Pmt: Plagiodiscus martensianus, Dka: Delphineis karstenii, Pfo: Pleurosigma formosum, Tpe: Triceratium pentacrinus, Cht: Cocconeis heteroidea, Psu: Paralia sulcata, Pgu: Podocystis guadalupensis. 
mediana e basal de exemplares coletados nas praias de Porto e Cagarras; 4) formado por Eunotogramma laeve, Lyrella lyra e Petroneis humerosa, presentes apenas na porção mediana dos exemplares coletados na praia do Porto, no mês de junho de 2007; 5) formado pelas espécies Cyclotella sp., Plagiogramma pulchellum e Terpsinoё mиsica, encontradas em todas as porções (apical, mediana e basal) dos exemplares coletados na praia do Porto; 6) formado por Biddulphia tridens e Podocystis spathulata, encontradas apenas na porção apical, em Cagarras (junho/2006); 7) formado por Plagiodiscus martensianus, Delphineis karstenii, Pleurosigma formosum e Triceratium pentacrinus, presentes nas porções mediana e basal, nos três locais de coleta, em de junho/2006; 8) formado por Cocconeis heteroidea, Paralia sulcata e Podocystis guadalupensis presentes apenas em exemplares coletados na praia do Porto, no mês de junho/2006 (Fig. 5, Tab. 1)

Os resultados obtidos confirmaram que Galaxaura rugosa transporta em seu talo várias espécies de diatomáceas epífitas contribuindo, assim, para o aumento da produtividade de zonas costeiras do Arquipélago de Fernando de Noronha, porém a fixação das espécies não ocorre de modo uniforme nos diversos pontos de coleta, nem nas diversas partes da macroalga.

\section{Agradecimentos}

Os autores agradecem à Coordenação de Aperfeiçoamento de Pessoal de Nível Superior (CAPES) e à Fundação de Amparo à Ciência e Tecnologia do Estado de Pernambuco (FACEPE), pela Bolsa de Mestrado concedida ao primeiro Autor; ao Conselho Nacional de Desenvolvimento Científico e Tecnológico (CNPq), no âmbito do Processo n. 477354/2004-1, pelo apoio financeiro recebido para a realização das coletas no Arquipélago de Fernando de Noronha.

\section{Referências bibliográficas}

Almeida, F.F.M. 2002. Arquipélago de Fernando de Noronha - Registro de monte vulcânico do Atlântico Sul. Pp. 361-368. In: C. Schobbenhaus; D.A. Campos; E.T. Queiroz; M. Winge \& M.L.C. Berbert-Born (eds.). Sítios Geológicos e Paleontológicos do Brasil. Brasília, DNPM/CPRM - Comissão Brasileira de Sítios Geológicos e Paleobiológicos (SIGEP).

Azevedo, A.C.G. 1999. Composição florística das diatomáceas (Bacillariophyta) epífitas em duas espécies de Bostrychia Montagne (Rhodophyta). Insula 28: 101-148.

Azevedo, A.C.G. \& Cutrim, M.V.J. 1999. Diatomáceas epífitas em Bostrychia Montagne (Rhodophyta) do manguezal da ilha de São Luís, Estado do Maranhão, Brasil: Naviculales e Bacillariales. Boletim do Laboratório de Hidrobiologia 12: 13-22.

Azevedo, A.C.G. \& Cutrim, M.V.J. 2000. Diatomáceas (Bacillariophyta) epífitas em Bostrychia Montagne (Rhodophyta) do manguezal da ilha de São Luís, Estado do Maranhão, Brasil: excluído Naviculales e Bacillariales. Boletim do Laboratório de Hidrobiologia 13: $1-17$.

Bergey, E.A.; Boettiger, C.A. \& Resh, V.H. 1995. Effects of water velocity on the architecture and epiphytes of Cladophora glomerata (Chlorophyta). Journal of Phycology 31: 264-271.

Buselato-Toniolli, T.C. 1986. Diatomoflórula (Bacillariophyceae) associada à Hypnea musciformis (Wulfen) Lamouroux (Rhodophyta) do litoral de Torres, Rio Grande do Sul, Brasil. Iheringia 35: $65-123$.
Cunha, M.C.C. \& Eskinazi-Leça, E. 2003. Estrutura da flora de diatomáceas epífitas em fanerógamas marinhas no litoral de Pernambuco. Pp. 384-385. In: V. Claudino-Sales; I. M. Tonini \& E.W.C. Dantas (eds.). Anais de Trabalhos Completos do VI Congresso de Ecologia do Brasil, Fortaleza, Sociedade Brasileira de Ecologia.

Cupp, E.E. 1943. Marine plankton diatoms of the west coast of North America. Bulletin of the Scripps Institution Oceanographic University Califonia 5: 1-238,

Edelstein, T. \& Komarovsky, B. 1961. Epiphytic algae on Halimeda tuna f. platydisca (Dec.) Bart. In Halfa bay. Bulletin of the Research Council Israel 10d: 54-58.

Edsbagge, H. 1968. The Composition of the epiphytic diatom flora and Swedish West Coast. Botanica Marina 11: 68-71.

Eskinazi-Leça, E.; Magalhães, K.M. \& Moura Junior, A.M. 2003. Variação quantitativa da diatomoflora epífita na fanerógama marinha Halodule wrightii Ascherson do litoral de Pernambuco. Pp. 270-271. In: V. Claudino-Sales; I.M. Tonini \& E.W.C. Dantas (eds.). Anais de Trabalhos Completos do VI Congresso de Ecologia do Brasil. Fortaleza, Sociedade Brasileira de Ecologia.

Eston, V.R.; Migotto, A.E.; Oliveira Filho, E.C.; Rodrigues, S.A. \& Freitas, J.C. 1986. Vertical distribution of benthic marine organisms on rocky coasts of the Fernando de Noronha archipelago (Brazil). Boletim do Instituto Oceanográfico 34: 37-53.

Ferrario, M.E.; Sar, E. \& Sala, S. 1995. Metodologia Básica para el studio del fitopláncton com especial referéncia a las diatomáceas. Pp. 1-23. In: K. Alveal; M.E. Ferrario; E.C. Oliveira \& E. Sar (eds.). Manual de Metodos Ficológicos. Concepcion, Universidad de Concepcion.

Ferreira, S. \& Seeliger, U. 1985. The colonization process of algal epiphytes on Ruppia Maritima Linnaeus. Botanica Marina 28: 245-249.

Frankovich, T.A.; Gaiser, E.E.; Zieman, J.C. \& Wachnicka, A.H. 2006. Spatial and temporal distributions of epiphytic diatoms growing on Thalassia testudinum Banks ex König: relationships to water quality. Hydrobiologia 569: 259-271.

Hustedt, F. 1930. Die Süsswasser-Flora Mitteleuropas. Heft 10: Bacillariophyta (Diatomeae). Jena.

Hustedt, F. 1937-1938. Systematische und Ökologische Untersuchungen über die Diatomeen-Flora von Java, Bali und Sumatra. Archiv für Hydrobiologie 15: 187-295.

Hustedt, F. 1961. Die Kieselalgen. L. Rabenhorst's KryptogamenFlora von Deutschland, Österreich und der Schweiz 7: 1-160.

Jaccard, P. 1901. Etude comparative de la distribution florale dans une portion des Alpes et du Jura. Bulletin de la Société Vaudoise de Sciences Naturelles 37: 547-579.

Kutner, M.B.B. 1961. Algumas diatomáceas encontradas sobre algas superiores. Boletim do Instituto Oceanográfico de São de Paulo 11: 3-11.

Laborel-Deguen, F. 1963. Nota preliminar sobre a ecologia das pradarias das fanerógamas marinhas nas costas dos Estados de Pernambuco e da Paraíba. Trabalhos do Instituto de Biologia Marítima e Oceanografia (3/4): 39-50.

Main, S.P. \& McIntire, C.D. 1974. The distribution of epiphytic diatoms in Yaquina estuary, Oregon (U.S.A.). Botanica Marina 17: $88-89$.

Mann, D.G. 1999. The species concept in diatoms. Phycologia 38: 437-495.

McIntire, C.D. \& Moore, W.W. 1977. Marine littoral diatoms: Ecological considerations. Pp. 333-371. In: D. Werner (ed.). The Biology of Diatoms, Oxford, University of California Press.

Moreira Filho, H. 1959. Diatomáceas do Paraná: a flora diatomológica do Sargassum. Boletim do Instituto de História Natural da Secretária de Agricultura 2: 1-27.

Moreira Filho, H. 1966. Contribuição ao estudo das Bacillariophyceae (Diatomáceas) no ágar-ágar (gelosa) e agarófitos. Boletim Botânico da Universidade Federal do Paraná 16: 1-55. 
Moreira Filho, H. \& Oliveira Filho, E.C. 1976. Diatomáceas epífitas em duas populações de Sargassum cymosum C. Agardh. Acta Biologica Paranaense 5: 53-75.

Moreira Filho, H.; Mattos, A. \& Valente-Moreira, I.M. 1977-1978. Diatomáceas epífitas em Codium decorticatum (Woodward) Howe. Tribuna Farmacêutica 45-46: 3-17.

Moreira Filho, H. \& Valente-Moreira, I.M. 1980. Diatomáceas epífitas em Ulva fasciata Delile. Boletim do Museu Botânico Municipal 41: 1-10.

Moreira Filho, H. \& Valente-Moreira, I.M. 1981. Avaliação taxonômica e ecológica das diatomáceas (Bacillariophyceae) epífitas em algas pluricelulares obtidas nos litorais dos Estados do Paraná, Santa Catarina e São Paulo. Boletim do Museu Botânico Municipal 47: 1-17.

Moreira Filho, H. \& Teixeira, C. 1963. Noções gerais sobre as diatomáceas (Chrysophyta - Bacillariophyceae). Boletim Botânico da Universidade Federal do Paraná 11: 1-26.

Moreno, J.L; Licea, S. \& Santoyo, H. 1996. Diatomeas del Golfo de Califórnia. 1 ed. Mexico. Universidad Autonoma de Baja California Sur, SEP-FOMES, PROMARCO.

Moura, A.N.; Passavante, J.Z.O. \& Silva-Cunha, M.G.G. 1993. Diatomáceas perifíticas fixas em substratos natural. Trabalhos Oceanográficos da Universidade Federal de Pernambuco 22: $34-86$.

Nagumo, T. \& Hara, Y. 1990. Species composition and vertical distribuition of diatoms occuring in a Japanese mangrove forest. Japan Journal Phycology 38: 333-343.

Navarro, J.N. 1982. Marine diatoms associated with mangrove prop roots in the Indian River, Florida, USA. Phycologia 61: $1-151$.

Navarro, J.N. 1983. A survey of the marine diatoms of Puerto Rico. Botanica Marina 26: 119-136.

Navarro, J.N.; Perez, C.; Arce, N. \& Arroyo, B. 1989. Benthic marine diatoms of Caja de Muertos Island, Puerto Rico. Nova Hedwigia 49: 333-367.

Pacobahyba, L.D.; Eskinazi-Leça, E.; Silva-Cunha, M.G.G. \& Koening, M.L. 1993. Diatomáceas (Bacillariophyceae) epífitas na fanerógama marinha Halodule wrightii Aschers coletada no ambiente costeiro de Itamaracá-PE. Trabalhos Oceanográficos da Universidade Federal de Pernambuco 22: 39-64.

Peragallo, H. \& Peragallo, M. 1897-1908. Diatoms marines de France et des districtes maritimes voisins. J. Tempere.

Pereira, S.M.B. 2006. Algas marinhas bentônicas do Arquipélago de Fernando de Noronha (PE) e Arquipélago de São Pedro e São Paulo. Pp. 113-126. In: R.J.V. Alves \& J.W.A. Castro (eds.). Ilhas Oceânicas Brasileiras da Pesquisa ao Manejo. Cap. 5, $2^{\mathrm{a}}$ parte. Brasília, Ministério do Meio Ambiente.

Ricard, M. 1987. Atlas Du Phytoplancton Marin: Diatomophycées. Paris, Editions Du Centre National de la Recherce Scientifique.

Rivera, P.R. 1973. Diatomaceas epifitas en Gracilaria verrucosa (Hudson) Papenfuss recoletada en la costa chilena. Gayana 25: $1-116$.
Rohlf, F.J. 2000. NTSYSpc, numerical taxonomy and multivariate data analysis system. Version 2.01. New York, Exeter Software.

Round, F.E.; Crawford, R.M. \& Mann, D.G. 1990. The Diatoms biology and morphology of the genera. Cambridge, Cambridge University Press.

Ruesink, J.L. 1998. Diatom epiphytes on Odonthalia floccosa: The importance of extent and timing. Journal of Phycology 34: 29-38.

Shannon, C.E. 1948. A mathematical theory of communication. Bulletin of System Tecnology Journal 27: 379-423.

Siqueiros-Beltrones, D.A. \& Castrejón, E.S. 1999. Structure of benthic diatom assemblages from a mangrove environment in a Mexican subtropical lagoon. Biotropica 31: 48-70.

Siqueiros-Beltrones, D.A.; Serviere-Zaragoza, E. \& Argumedo Hernández, U. 2002. Epiphytic diatoms of Macrocystis pyrifera (Linnaeus) C. Agardh from the Baja California Peninsula, Mexico. Oceánides 17: 31-39.

Siqueiros-Beltrones, D.A; López-Fuerte, F.O. \& Gárate-Lizárragua, I. 2005. Structure of diatom assemblages living on prop roots of the red mangrove (Rhizophora mangle Linnaeus) from the West Coast of Baja California Sur, México. Pacific Science 59: 79-96

Takano, H. 1961. Epiphytic upon japanese agar seaweeds. Bulletin of the Tokai Regional Fisheries Research Laboratory 31: 269-274.

Takano, H. 1962. Notes on Epiphytic Diatom upon Seaweeds from Japan. Journal of the Oceanographical Society of Japan 18: 29-33.

Teixeira, W.; Cordani, U. \& Menor, E.A. 2003. Caminhos do Tempo Geológico. Pp. 26-63. In: R. Linsker (ed.). Arquipélago de Fernando de Noronha o Paraíso do Vulcão. São Paulo, Terra Virgem Editora.

Valente-Moreira, I.M. \& Moreira Filho, H. 1980. Diatomáceas epífitas em Padina vickersiae Hovt ex Howe. Tribuna Farmacêutica 48: 114-122.

Van Heurk, H. 1896. A Treatise on the Diatomaceae. London, William Wesley \& Son.

Villaça, R.; Pedrini, A.G.; Pereira, S.M.B. \& Figueiredo, M.A.O. 2006. Flora Marinha Bentônica das Ilhas Oceânicas Brasileiras. Pp. 105-146. In: R.J.V. Alves \& J.W.A. Castro (eds.). Ilhas Oceânicas Brasileiras da Pesquisa ao Manejo. Brasília, MMA-SBF.

Virnstein, R.W. 1987. Seagrass - associated invertebrate communities of the southeastern U.S.A: A review symposium on subtropicaltropical seagrass of southeastern United State. Marine Research Publications 42: 89-116.

Wah, T.T. \& Wee, Y.C. 1988. Diatoms from mangrove environments of Singapore e southern peninsular Malasia. Botanica Marina 31: $317-327$.

Wetherbee, R.; Lind, L.J.; Burke, J. \& Quatrano, S.R. 1998. The first kiss: establishment and control of initial adhesion by raphid diatoms. Journal Phycology 34: 9-15.

Zar, J.H. 1999. Biostatiscal Analyses. 4 ed. New Jersey, Prentice Hall.

Versão eletrônica do artigo em www.scielo.br/abb e http://www.botanica.org.br/acta/ojs 